\title{
Erratum to: Analytical and Computational Methods in Probability Theory
}

\author{
Vladimir V. Rykov ${ }^{1(\bowtie)}$, Nozer D. Singpurwalla ${ }^{2}$ (D), \\ and Andrey M. Zubkov ${ }^{3}$ \\ 1 Department of Applied Probability and Informatics, RUDN University, \\ Moscow, Russia \\ vladimir_rykov@mail.ru \\ ${ }^{2}$ City University of Hong Kong, Hong Kong, Hong Kong \\ ${ }^{3}$ Steklov Mathematical Institute of Russian Academy of Sciences, \\ Moscow, Russia
}

\section{Erratum to: \\ V. V. Rykov et al. (Eds.): \\ Analytical and Computational Methods in Probability Theory, LNCS 10684, https://doi.org/10.1007/978-3-319-71504-9}

In an earlier version of these proceedings, the affiliations of the volume editors Vladimir V. Rykov and Andrey M. Zubkov were incorrect. This has been corrected. Vladimir V. Rykov Department of Applied Probability and Informatics, RUDN University, Moscow, Russia

Andrey M. Zubkov

Steklov Mathematical Institute of Russian Academy of Sciences, Moscow, Russia

The updated online version of this book can be found at https://doi.org/10.1007/978-3-319-71504-9 